\title{
Aquatic treadmill running reduces muscle soreness following intense sprint exercise in trained men
}

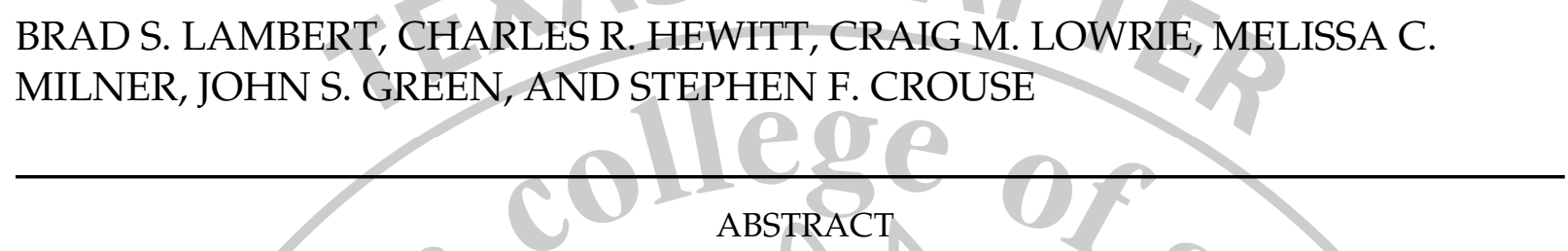

Delayed onset muscle soreness is associated with muscle damage, disturbances in proprioception, and decreases in muscular power. The purpose was to determine if short duration aquatic treadmill (ATM) running reduces muscle soreness following intense sprint exercise in trained men. Twenty trained men $(180.3 \pm 4.4 \mathrm{~cm}, 86.3 \pm 5.8 \mathrm{~kg}, 20 \pm 1 \mathrm{yr})$ were recruited and randomly divided into two groups: ATM recovery (ATMRec) and passive recovery (PRec). During testing, subjects performed a warm-up followed by sixteen $110 y$ rd cutback runs with a sprint of $60 y$ rds, sharp change of direction, and a return sprint of $50 y$ rds. Work to rest ratio was set at 1:3. Additionally, following exercise, the ATMRec group performed ATM running using a HydroWor ${ }^{\circledR}$ treadmill at $5 \mathrm{mph}, 50 \%$ maximal jet resistance, and water $\left(33^{\circ} \mathrm{C}\right)$ level at chest depth for 10min. Both groups then evaluated their level of soreness/pain using a numerical rating scale (NRS: $0-10,0=$ no pain, $10=$ worst pain) immediately following all exercise (IPE), $24 \mathrm{~h}$, and $48 \mathrm{~h}$ post exercise in the following regions: ARMS, LEGS, BACK, CHEST, SHOULDERS, HIPS, ABDOMEN, NECK, OVERALL. Data were analyzed for group x time interactions using a 2x3 Generalized Linear Mixed Model for non-parametric data $(\alpha \leq 0.05)$. For significant interactions, the same procedure was used to analyze between group differences at the same measurement timepoint $(\alpha \leq 0.05)$.

\begin{tabular}{|l|llll|}
\hline Independent Var. & Group & IPE & $\mathbf{2 4 h}$ & $\mathbf{4 8 h}$ \\
\hline \multirow{2}{*}{ LEGS } & ATMRec & $3.3 \pm 0.3^{+}$ & $3.7^{+} 0.4^{+}$ & $3.2 \pm 0.7$ \\
& PRec & $4.5 \pm 0.7^{\mathrm{a}}$ & $5.2 \pm 0.5^{\mathrm{a}}$ & $3.3 \pm 0.4^{\mathrm{b}}$ \\
\hline \multirow{2}{*}{ BACK } & ATMRec & $1.3 \pm 0.4^{\mathrm{a}}$ & $0.9 \pm 0.3^{\mathrm{a}}$ & $0.5 \pm 0.3^{\mathrm{b}+}$ \\
& PRec & $2.5 \pm 0.9$ & $2.7 \pm 0.7$ & $1.8 \pm 0.6$ \\
\hline \multirow{2}{*}{ HIPS } & ATMRec & $1.2 \pm 0.4^{\mathrm{a}}$ & $2.1 \pm 0.5^{\mathrm{b}}$ & $1.3 \pm 0.4^{\mathrm{a}}$ \\
& PRec & $2.5 \pm 0.7^{\mathrm{a}}$ & $2.6 \pm 0.5^{\mathrm{a}}$ & $1.2 \pm 0.3^{\mathrm{b}}$ \\
\hline \multirow{2}{*}{ ABDOMEN } & ATMRec & $0.8 \pm 0.4^{+}$ & $1.1 \pm 0.3^{+}$ & $0.8 \pm 0.2$ \\
& PRec & $2.3 \pm 0.5^{\mathrm{a}}$ & $2.6 \pm 0.6^{\mathrm{a}}$ & $1.4 \pm 0.4^{\mathrm{b}}$ \\
\hline \multirow{2}{*}{ OVERALL } & ATMRec & $2.0 \pm 0.3^{\mathrm{a}}$ & $3.0 \pm 0.4^{\mathrm{b}}$ & $1.9 \pm 0.5^{\mathrm{a}}$ \\
& PRec & $3.2 \pm 0.5^{\mathrm{a}}$ & $3.5 \pm 0.4^{\mathrm{a}}$ & $2.1 \pm 0.3^{\mathrm{b}}$ \\
\hline
\end{tabular}

Values are means \pm SE for NRS scores $(0-10,0=$ no pain, $10=$ worst pain). Letter superscript $=$ sig. diff. $\mathrm{w} /$ in groups across time,$t=$ sig. diff. between groups at same measurement time point (comparisonwise $\alpha=0.05)$.

Aquatic treadmill running significantly reduces perceived muscle soreness and may enhance recovery following intense sprint exercise in trained men.

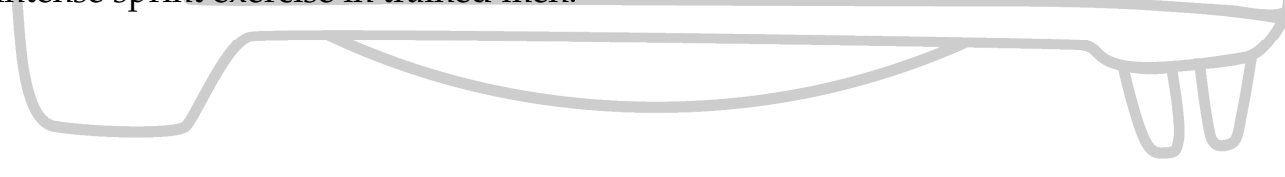

